\title{
In vivo assessment of secondary caries and dentin characteristics after traditional amalgam restorations
}

Isauremi Vieira de Assunção Pinheiro'

Boniek Castillo Dutra Borges ${ }^{1}$

Kenio Costa de Lima'

\begin{abstract}
Objective: This study aimed to evaluate in vivo the occurrence of secondary caries and dentin characteristics in permanent molars after traditional amalgam restorations, by means of clinical visual examination, radiographs and laser-induced fluorescence (LF) (DIAGNOdent).

Methods: Thirty first permanent molars of 30 schoolchildren in the 7 to 14 year-old age group were included. Caries was removed by hand. Thus, indirect pulp capping was performed with glassionomer cement (GIC), the cavity was varnished and amalgam filled. LF was measured before and after cavity preparation and after a 12-month observation period. Dentin color after cavity preparation and after the 12-month observation period was recorded. Recurrent caries was also investigated by visual clinical and radiographic examinations, in addition to dentin thickness between pulp and indirect GIC pulp capping. Data was analyzed by ANOVA for repeated measurements, paired " $t$ " test and descriptive statistic.

Results: There were statistically significant differences $(P<.05)$ among LF scores for dentin in all periods evaluated, with the lowest scores shown after 12 month of observation. There was no statistical difference between dentin color after cavity preparation and following 12 months of observation. Moreover, there was no recurrent caries attack at 12-month follow-up; dentin thickness between pulp and indirect GIC pulp capping was similar between baseline and final observation periods. It was concluded that the clinical restorative procedure using hand caries removal, indirect pulp capping with GIC, varnishing and amalgam filling the cavity did not provide secondary caries and increased dentin mineral content after 12 months. (Eur J Dent 2012;6:263-269)
\end{abstract}

Key words: Clinical trial; glass ionomer cement; remineralization; DIAGNOdent, recurrent caries

1 Department of Dentistry, Federal University of Rio Grande do Norte, Natal/RN, BRAZIL.

- Corresponding author:

Dr. Isauremi Vieira de Assunção Pinheiro

Department of Dentistry, Federal University of Rio Grande do Norte, Av Senador Salgado Filho, 1787,

Zip-Code: 59.056-000 Natal/RN, Brazil

Tel/Fax: +55 8432154135

Email: professorisauremidgmail.com

\section{INTRODUCTION}

Nowadays, cavity preparation should be performed with minimal tissue removal, since this conservation of sound tissue promotes the longevity of the restored tooth. ${ }^{1,2}$ Moreover, there is a general consensus for caries-affected dentin to be preserved during caries excavation because it can be remineralized. ${ }^{3-5}$ 
Traditionally, carious dentin may be removed mechanically with burs or hand excavators, and caries indicator dyes were developed to make operative caries removal a more objective., ${ }^{6,7}$ Since the use of diamond and tungsten carbide burs could result in indiscriminate caries removal, caries-infected and caries-affected dentin can be removed simultaneously, with possible extension into the underlying sound dentin. ${ }^{2}$ Moreover, evidence shows that there is a significant risk of over-extensive clinical cavity preparation by using a caries indicator during caries removal. ${ }^{8}$ Thus, both methods using burs or caries indicator dyes for caries excavation are not clinically encouraged given that they are contrary to the minimal intervention approach to dentistry.

Instead, manual carious dentin removal with sharp hand instruments, without using caries indicator dyes may be encouraged, because heavily infected soft and necrotic dentin must be removed before placing a restoration. ${ }^{9}$ This method is still controversial since there is difficulty in detecting infected, carious dentin accurately and reliably by visual and tactile clinical examination. ${ }^{10,11}$ However, it has been demonstrated that even without completely removing the infected dentin, the filled tooth did not show caries progression. ${ }^{12,13}$ Thus, the infected dentin that possibly remained after mechanical caries removal by hand could not have influenced caries progression. So, it is important to evaluate the tooth response after removing dentin caries by hardness criteria, since manual caries removal using sharp hand instruments without caries indicator dyes would preserve a larger amount of dentinal tissue. Furthermore, the placement of liners with cariostatic behavior, especially due to fluoride release, such as GIC on dentin after manual caries removal, could increase resistance to subsequent acid challenge. ${ }^{14}$

After GIC liner placement, a cavity varnish application was traditionally indicated in order to reduce microleakage. ${ }^{15}$ In vitro studies have contraindicated the clinical use of cavity varnish since it increases marginal microleakage, which is related to secondary caries. ${ }^{16,17}$ Nevertheless, laboratory studies are limited and do not reproduce the oral environment.

Long-term in vivo follow-up investigations are needed in an endeavor to evaluate dentinal reaction to manual caries removal according to hard- ness, GIC liner, and cavity varnish under amalgam fillings. Thus, the aim of this study was to evaluate in vivo the occurrence of secondary caries and dentin characteristics in permanent molars by means of visual examination, radiographs and laser fluorescence (DIAGNOdent) after restoring teeth using these clinical steps: hand caries removal, indirect pulp capping with GIC, applying copal varnish and filling the cavity with amalgam. The hypothesis tested was that the above mentioned protocol would product satisfactory results at the 12-month follow-up period.

\section{MATERIAL AND METHODS}

\section{Ethical Considerations}

The project was approved by the Research Ethics Committee of the local institution. All patients or their parents/guardians received information about the study and signed a free informed term of consent in accordance with Resolution 196/6 of the National Health Council and the Declaration of Helsinki (2000).

\section{Experimental design}

The factor under study in this prospective controlled clinical trial was the clinical protocol of amalgam filling after manual caries removal according to hardness, indirect pulp capping with GIC and cavity varnish application. The response variables were: (1) mineral content of dentin before and after cavity preparation and after a 12-month period of observation; (2) dentin color after cavity preparation and after 12 months; (3) recurrent caries attack after 12 months by radiographic examination; (4) presence of active soft carious dentin after 12 months by visual analysis; (5) thickness of dentin between the pulp and the GIC liner after baseline restoration and after 12 months by radiographic examination. Materials used in this study are listed in Table 1.

\section{Study population}

The sample consisted of 30 high caries-risk volunteers (chosen according to the Thompson and Kaim $^{18}$ criterial in the 7 to14 year-old age group who had not used any antibiotics during the period of 2 months prior to the study; whose teeth were examined and 30 of their permanent molars were selected for inclusion in the study. Inclusion criteria were: (1) teeth presenting cavitated caries 
lesions by visual examination; and (2) caries depth between the enamel-dentin junction and middle one-third of dentin shown in a radiograph. Exclusion criteria were the presence of restorations and white spot lesions or cavitations on other tooth surfaces.

Both the visual and radiographic exams were performed by a single trained and calibrated operator. Visual examination was made after pumice and water prophylaxis, using a flat mirror (SSWhite, Rio de Janeiro, RJ, Brazil), triplex syringe and headlight (Gnatus, Araraquara, SP, Brazil). For radiographic examination, bite-wing radiography was performed using a positioner (Indusbello, Londrina, PR, Brazil) to visualize the carious lesion depth.

\section{Clinical Steps (Baseline Period)}

After anesthesia and rubber dam placement, access to caries was created with a carbide bur \#245 (KG Sorensen, Barueri, SP Brazil). DIAGNOdent was calibrated with a ceramic standard using a fiber-optic broad tip (tip B) and fluorescence reading was taken on carious dentin before cavity preparation. Tip B was used around the entire extension of the cavosurface angle three times. The

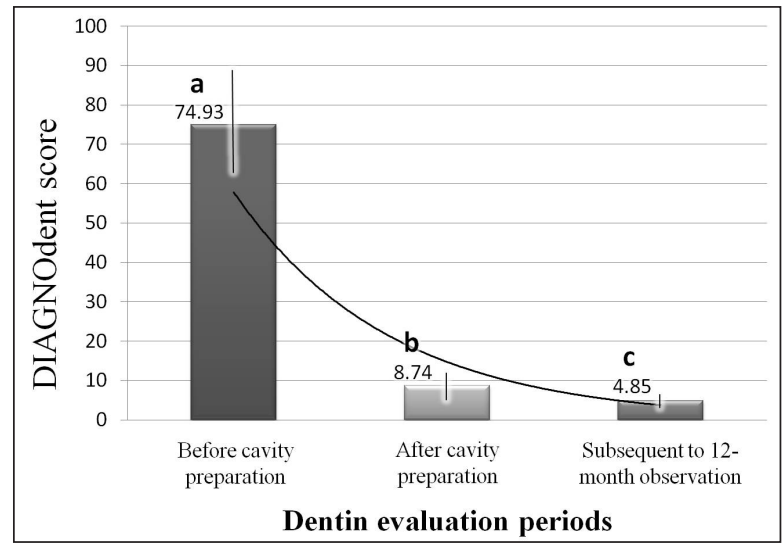

Figure 1. Demineralization of dentin during observation periods in this study. Means followed by different lowercase letters are statistically different $(P<.05)$.

Table 1. Materials used in this study.

\begin{tabular}{lcc}
\hline Commercial number (manufacturer) & Classification & Composition \\
\hline $\begin{array}{l}\text { Vidrion F } \\
\text { SS White, Rio de Janeiro, RJ, Brazil }\end{array}$ & $\begin{array}{c}\text { Conventional low-viscosity glass } \\
\text { ionomer cement }\end{array}$ & $\begin{array}{c}\text { Powder: Na-Ca-Al-flurosilicate-Ba sulfate, } \\
\text { acrylic acid; liquid: tartaric acid, water }\end{array}$ \\
$\begin{array}{l}\text { Cavitine } \\
\text { SS White, Rio de Janeiro, RJ, Brazil }\end{array}$ & Copal cavity varnish & Nitrocellulose (8g\%), ethyl acetate and ethylic \\
Velvalloy & Admixed amalgam & Silver $(70 \mathrm{~g} \%)$, stantium $(26 \mathrm{~g} \%)$, copper $(3 \mathrm{~g} \%)$ \\
SS White, Rio de Janeiro, RJ, Brazil & and zinc $(1 \mathrm{~g} \%)$ \\
K-Dent & Mercury & Mercury \\
Quimidrol, Joinville, SC, Brazil & & 7 \\
\hline
\end{tabular}

peak of each reading was recorded and a mean was obtained.

Manual caries removal was performed with a sharp excavator (SS White), so that the operator removed all soft dentin until a slightly hard surface was tactually perceived. ${ }^{24}$ After carious removal, the color of the remaining pulp floor dentin was assessed by a previously calibrated examiner (kappa 0,93) according to the following scores: ${ }^{19}$ (0) light yellow, (1) yellow, (2) light brown, (3) dark brown, (4) Black. Afterwards, the fluorescence of dentin was again measured using the DIAGNOdent device with the tip $B$ set to $1 \mathrm{~mm}$ from the pulp cavity wall. Cavity finishing was performed with a carbide bur \#245 (KG Sorensen) coupled to a lowspeed turbine (Kavo). The cavity was washed with saline solution and dried using sterile cotton wool.

GIC (Vidrion F, SS White) was used to place indirect pulp capping (about $1 \mathrm{~mm}$ thickness) on the cavity floor after agglutination of this material according to the manufacturer's recommendation. Two thin coats of cavity varnish Cavitine ISS White) were applied on the entire cavity and dried with a short, gentle blast of air after each application. After this, teeth were restored with Velvalloy (SS White) associated with K-Dent Mercury (Quimidrol) in a 1:1 ratio. Occlusal contacts were verified with a carbon paper (Accu Film II, Parkell In., Farmingdale, NY, USAl and adjusted if necessary using 12-blade finishing burs (Maillefer, Ballaigues, Switzerland). Forty-eight hours after amalgam condensation, amalgams were finished and polished using 12-blade burs (Maillefer) or abrasive rubbers (KG Sorensen), respectively.

After the teeth were restored, a bitewing radiograph was taken for the purpose of comparing the teeth after the 12-month follow-up period. To obtain geometric standardization of films, bitewing film holders were used. A piece of self-cured acrylic resin (Duralay, Reliance, Illinois, USA) was 
placed on the film holder (Jon, São Paulo, SP, Brazil) which was then placed on the occlusal surface of the experimental tooth and its antagonist to form an impression of the anatomy of this surface. It was, therefore, possible to relocate the device in the same position in the different experimental periods.20 Kodak E-Speed films (Eastman Kodak Co., New York, USA, lot number 3107662) were used and radiographs were taken using the same mobile X-ray source (Timex 70C mobile column, Gnatus; exposure time: $0.64 \mathrm{~s}$ ). The radiographs were processed manually in Kodak developing (lot number $0637 \mathrm{C6}$ 02687) and fixing (lot number 0714 B6 02251) solutions using the following temperature/time combination: $2 \mathrm{~min}$ in the developer and $4 \mathrm{~min}$ in the fixer at a temperature of $26^{\circ} \mathrm{C}$. All procedures were performed by a single professional.

\section{Monitoring the Restorations}

After the 12-month follow-up period, all individuals were scheduled for re-evaluation. Clinical examination executed and a bitewing radiograph was taken using the same film holder of the impression initially defined. Similar X-ray source and film processing conditions as those used at baseline were applied.

The teeth were anesthetized and a rubber dam was placed. Amalgam and GIC were removed by the same operator, using a \#245 carbide bur (KG Sorensen) in order to assess the dentin underneath these materials. This direct visualization is the gold-standard method for evaluating recurrent caries (soft dentin) and dentin color visually, and mineral content by DIAGNOdent. The presence of soft dentin (typical in active recurrent caries) was observed by clinical examination. Moreover, dentin color at the pulp wall was recorded using the previously described scores19 and a laser-induced fluorescence reading with DIAGNOdent was performed under similar conditions to those used at baseline. After this, tooth was refilled using clinical steps similar to those used at baseline.

Baseline and 12-month (final) radiographs were codified and analyzed in a dark room. A single calibrated examiner (kappa 0,91) performed this exam with a negatoscope, a 2x magnifying glass (Maped do Brasil, São Paulo, SP, Brazil) and a millimeter ruler (Prisma, São Paulo, SP, Brazil).
The presence of a radiolucent area surrounding the restoration margins lindicative of secondary caries) was observed in the final radiograph. In addition, the dentin thickness between the pulp and GIC liner was recorded in both radiographs (baseline and final).

\section{Statistical Analysis}

Analysis of variance (ANOVA) for repeated measurements and Tukey test were performed to compare the amount of mineral in dentin before and after cavity preparation and after the 12-month observation period. Dentin color after cavity preparation and after 12 months was compared by means of the paired Student's-t test. Spearman's correlation test was performed to associate dentin color with the amount of mineral in dentin. All the above-mentioned tests were performed at a significance level of $5 \%$. Descriptive statistics was performed to characterize recurrent caries attack after 12 months by either clinical visual or radiographic examination. Dentin thicknesses were compared using descriptive statistics.

\section{RESULTS}

\section{Dentin Mineral Content}

Means and standard deviations emitted by the DIAGNOdent device are shown in Figure 1. There was statistically difference between mineral content of dentin in all evaluated periods $(P<.001)$, showing the highest value before caries removal and the lowest after 12 months.

\section{Dentin Color}

There was no statistical difference ( $P=$.07) between dentin color means after cavity preparation $(1.73 \pm 0.64)$ and after 12 months $(1.9 \pm 0.66)$.

Dentin Color versus Dentin Mineral Content

There was no correlation between dentin color and dentin mineral content $\left(P=.74 ; r^{2}=-0.33\right)$.

\section{Recurrent caries}

After 12 months, no recurrent caries was observed either by clinical visual or radiographic examinations.

\section{Remaining Dentin Thickness}

There was no increase or decrease in remaining dentin thickness after 12 months when compared with the baseline observation $11.72 \mathrm{~mm} \pm$ $0.49 \mathrm{~mm}$ ). 


\section{DISCUSSION}

The hypothesis tested in this study was validated. Results obtained showed that the traditional amalgam filling protocol is effective, since no secondary caries was observed by clinical and radiograph criteria, besides a progressive remineralization in dentin was detected by DIAGNOdent readings after the 12 -month follow-up period.

DIAGNOdent is a laser fluorescence device able to capture the fluorescence emitted from dental tissues when teeth are stimulated by its diode laser with a wavelength of $655 \mathrm{~nm} .{ }^{21}$ The tooth surface is illuminated by a red light (modulated excitation wavelength at $655 \mathrm{~nm}$ ), which is produced by a laser diode and transmitted by an optical fiber. The laser induces fluorescence in the mineralized tooth tissues, at a greater intensity in carious than in sound tooth tissues, which is then transmitted by an optical fiber to a photodiode with a long pass filter (wavelength $>680 \mathrm{~nm}$ ) in the detection device, so that changes in the fluorescence intensity are numerically quantified and translated to values ranging from 0 to $99 .{ }^{22}$ Since the scores emitted by DIAGNOdent showed a decrease at the 12-month evaluation, dentin remineralization occurred.

Dentin color and DIAGNOdent scores were assessed to avoid influences from possible amalgam staining not visually perceived in cavity walls. Although clinicians have correlated dentin darkening with its high mineral content, in this study no correlation was shown between dentinal colors and DIAGNOdent scores, and no statistically significant darkening was shown after 12-months of observation. This finding presupposes that dentin color may not depend only on the mineral content, but also on other variables, such intrinsic characteristics of collagen fibrils and staining substances released from microorganisms. However, further researches are needed to confirm this theory.

Remineralization and even hypermineralization of demineralized dentin has been demonstrated by various GICs in vitro ${ }^{23}$ and in situ. ${ }^{24}$ High concentrations of fluoride and strontium ions have been found in dentin beneath GIC. ${ }^{9}$ Mineral re-deposition into demineralized dentin lesions has exhibited higher resistance to the second acid challenge, for example, restoration leakage and recurrent caries attack. ${ }^{25}$ Thus, the increase in mineral content achieved in dentin after the 12-month observation period was possibly composed of ions, such as fluoride and strontium, released from the GIC.

For remineralization to be effective, the freshly mixed GIC must be closely adapted to the cariesaffected dentin. This allows the relatively high initial release of fluoride and other ions from the cement to augment physiological remineralization and the relatively low initial $\mathrm{pH}$ to reduce the numbers and viability of the cariogenic microorganisms present. ${ }^{9}$ Although the infected dentin was manually removed during cavity preparation, a certain amount of bacteria still remain. ${ }^{26}$ The fluoride release by $\mathrm{GIC}_{1}{ }^{27}$ in addition to maintaining a seal at the cavosurface restoration margin, could inhibit residual bacteria proliferation, ${ }^{2}$ and prevent secondary caries. Since indirect pulp capping was placed on the cavity floor of middle-third cavities in this investigation, GIC was not able to stimulate the pulp to produce a mineral barrier after the 12-month follow-up, which would increase the distance between pulp and GIC.

Although in vitro studies ${ }^{16,17}$ have shown increased microleakage in amalgam restorations under cavity varnish due to dissolution of the varnish before the formation of corrosion products, this in vivo investigation demonstrated that the clinical relevance of laboratory studies is questionable. Results of the present study with regard to secondary caries probably indicate the ability of corrosion products to form at the interface between tooth and restoration, which could help to protect it against penetration of oral fluids and cariogenic microorganisms. It is important to highlight that admixed alloys produce corrosion products faster than unicompositional alloys. ${ }^{28}$ Since the Velvalloy amalgam used in this study is an admixed alloy, corrosion products were probably produced before varnish dissolution.

Adhesive dentistry has focused on improvements of composite resins and adhesive systems, since composite restoration have become popular because of their esthetic appeal. ${ }^{29}$ Nevertheless, amalgam is still extensively used worldwide, ${ }^{30}$ mainly in public health care services which attend low-income, high caries risk patients. In the actual dentistry, the minimally invasive dentistry approach has gained great attention worldwide. ${ }^{31}$ Therefore, this in vivo study showed that conventional tooth restorations with amalgam preparing the cavity by a minimal tissue removal can be effectively made. 


\section{CONCLUSION}

The restorative clinical procedure following hand caries removal, indirect pulp capping with GIC, varnishing and amalgam filling the cavity did not provide secondary caries and increased dentin mineral content after 12 months.

\section{REFERENCES}

1. Dennison JB, Hamilton JC. Treatment decisions and conservation of tooth structure. Dent Ciln N Am 2005;49:825845.

2. Silva NR, Carvalho RM, Pegoraro LF, Tay FR, Thompson VP. Evaluation of a self-limiting concept in dentinal caries removal. J Dent Res 2006;85:282-286.

3. ten Cate JM. Remineralization of caries lesions extending into dentin. J Dent Res 2001;80:1407-1411.

4. McComb D. Systematic review of conservative operative caries management strategies. J Dent Educ 2001;65:11541161.

5. Kidd EA. How 'clean' must a cavity be before restoration? Caries Res 2004;38:305-313.

6. Sato Y, Fusayama T. Removal of dentin by fuchsin staining. J Dent Res 1976;55:678-683.

7. Krause F, Braun A, Eberhard J, Jepsen S. Laser fluorescence measurements compared to electrical resistance of residual dentine in excavated cavities in vivo. Caries Res 2007; $41: 135-140$.

8. Banerjee A, Kidd EA, Watson TF. In vitro validation of carious dentin removed using different excavation criteria. Am J Dent 2003;16:228-230.

9. Smales RJ, Ngo HC, Yip KHK, Yu C. Clinical effects of glass ionomer restorations on residual carious dentin in primary molars. Am J Dent 2005;18:188-192.

10. Thylstrup A, Fejerskov O. Textbook of clinical cariology. Copenhagen, Munksgaard 1994.

11. Willmott NS, Wong FS, Davis GR. An X-ray microtomography study on the mineral concentration of carious dentine removed during cavity preparation in deciduous molars. Car Res 2007;41:129-134.

12. Mertz-Fairhurst EJ, Curtis Jr. JW, Ergle JW, Rueggeberg FA, Adair SM. Ultraconservative and cariostatic sealed restorations: results at year 10. J Am Dent Assoc 1998;129:5566.

13. Alves LS, Fontanella V, Damo AC, Ferreira de Oliveira E, Maltz M. Qualitative and quantitative radiographic assessment of sealed carious dentin: a 10-year prospective study. Oral Surg Oral Med Oral Pathol Oral Radiol Endod 2010;109:135-141.
14. Silveira AD, Borges BC, Almeida HV, Lima KC, Pinheiro IV. Progression of Non-Cavitated Lesions in Dentin through a Nonsurgical Approach: A Preliminary 12-Month Clinical Observation. Eur J Dent 2012;6:34-42.

15. Cenci MS, Piva E, Potrich F, Formolo E, Demarco FF, Powers JM. Microleakage in bonded amalgam restorations using different adhesive materials. Braz Dent J 2004;15:13-18.

16. Vermeesch G, Leloup G, Delmee M, Vreven J. Antibacterial activity of glass-ionomer cements, compomers and resin composites: relatioship between acidity and material setting phase. J Oral Rehabil 2005;32:368-374.

17. da Silva AF, Piva E, Demarco FF, Correr-Sobrinho L, Osinga PW. Microleakage in conventional and bonded amalgam restorations: influence of cavity volume. Oper Dent 2006;31:377-383.

18. Thompson VP, Kaim JM. Nonsurgical treatment of incipient and hidden caries. Dent Clin N Am 2005;49:905-921.

19. Bjorndal L, Larsen T, Thylstrup A. A clinical and microbiological study of deep carious lesions during stepwise excavation using long treatment intervals. Caries Res 1997;31:411-417.

20. Oliveira EF, Carminatti G, Fontanella V, Maltz M. The monitoring of deep caries lesions after incomplete dentine caries removal: results after 14-18 months. Clin Oral Invest 2006;10:134-139.

21. Hibst R, Paulus R, Lussi A. Detection of occlusal caries by laser luorescence. Basic and clinical investigations. Med Laser Appl 2001;16:205-213.

22. Chu CH, Lo EC, You DS. Clinical diagnosis of fissure caries with conventional and laser-induced fluorescence techniques. Lasers Med Sci 2010;25:355-362.

23. Kitasako Y, Nakajima M, Foxton RM, Aoki K, Pereira PN, Tagami J. Physiological remineralization of artificially demineralized dentin beneath glass ionomer cements with and without bacterial contamination in vivo. Oper Dent 2003;28:274-280.

24. ten Cate, van Duinen RN. Hypermineralization of dentinal lesions adjacent to glass-ionomer cement restorations. $J$ Dent Res 1995;74:1266-1271.

25. Tantbirojn D, Feigal RJ, Ko CC, Versluis A. Remineralized dentin lesions induced by glass ionomer demonstrate increased resistance to subsequent acid challenge. Quintessence Int 2006;37:273-281.

26. Pinheiro IV, Borges BC, Colombo AP, Lima KC. Clinical and microbiological characteristics in predicting dentine caries progression. Acta Odontol Latinoam 2009;22:143-149.

27. Silveira AD, Borges BC, Almeida HV, Lima KC, Pinheiro IV. Progression of non-cavitated lesions in dentin through a nonsurgical approach: a preliminary 12-month clinical observation. Eur J Dent 2011;5:229-236. 
28. Gallato A, Angnes G, Reis A, Loguercio AD. Long-term monitoring of microleakage of different amalgams with different liners. J Prosthet Dent 2005;93:571-576.

29. Cunha LG, Alonso RC, Souza-Júnior EJ, Neves AC, CorrerSobrinho L, Sinhoreti MA. Influence of the curing method on the post-polymerization shrinkage stress of a composite resin. J Appl Oral Sci 2008;16:266-270.

30. Christensen GJ. Longevity of posterior tooth dental restorations. J Am Dent Assoc 2005;136:201-203.

31. Borges BC, Borges JS, Araújo LS, Machado CT, dos Santos AJ, Pinheiro IV. Update on nonsurgical, ultraconservative approaches to treat effectively non-cavitated caries lesions in permanent teeth. Eur J Dent 2011;5:229-236. 